PROCEEDINGS OF THE

AMERICAN MATHEMATICAL SOCIETY

Volume 137, Number 2, February 2009, Pages 695-698

S 0002-9939(08)09714-1

Article electronically published on September 12, 2008

\title{
DIMENSION REDUCTION FOR HYPERBOLIC SPACE
}

\author{
ITAI BENJAMINI AND YURY MAKARYCHEV
}

(Communicated by Mario Bonk)

\begin{abstract}
A dimension reduction for hyperbolic space is established. When points are far apart, an embedding with bounded distortion into $H^{2}$ is achieved.
\end{abstract}

\section{INTRODUCTION}

Dimension reduction results for Euclidean spaces have numerous applications in Theoretical Computer Science. They help to significantly reduce the space required for storing multidimensional data, and thus to improve performance of many algorithms. In this paper, we prove a dimension reduction theorem for hyperbolic space. Our result shows that many existing algorithms for Euclidean spaces that rely on dimension reduction can also be applied to hyperbolic spaces.

We refer the reader to a paper of Ailon and Chazelle [1] for background on dimension reduction algorithms and some of their applications. We also refer the reader to a paper of Krauthgamer and Lee [5, which studies combinatorial algorithms for hyperbolic spaces. For background on hyperbolic geometry see e.g. 3 .

1.1. Our results. In this paper, we consider the Poincaré half-space model of hyperbolic space $H^{n}$. Recall that every point is represented as a pair $(z, x), z \in \mathbb{R}^{+}$, $x \in \mathbb{R}^{n-1}$ in this model. The distance between two points $p_{1}=\left(z_{1}, x_{1}\right)$ and $p_{2}=\left(z_{2}, x_{2}\right)$ is defined by

$$
d\left(p_{1}, p_{2}\right)=\operatorname{arccosh}\left(1+\frac{\left\|x_{1}-x_{2}\right\|^{2}+\left(z_{1}-z_{2}\right)^{2}}{2 z_{1} z_{2}}\right) .
$$

For brevity, we define $F\left(r, z_{1}, z_{2}\right)$ as follows:

$$
F_{z_{1}, z_{2}}(r)=\operatorname{arccosh}\left(1+\frac{r^{2}+\left(z_{1}-z_{2}\right)^{2}}{2 z_{1} z_{2}}\right) \text {. }
$$

Then

$$
d\left(p_{1}, p_{2}\right)=F_{z_{1}, z_{2}}\left(\left\|x_{1}-x_{2}\right\|\right) .
$$

Suppose we are given an $n$-point subset $S$ of hyperbolic space. Let $T$ be its projection on $\mathbb{R}^{n-1}$ :

$$
T=\{x:(z, x) \in S\} .
$$

By the Johnson-Lindenstrauss lemma [4, there exists an embedding of $T$ into an $O\left((\log n) / \varepsilon^{2}\right)$ dimensional Euclidean space such that for every $x_{1}, x_{2} \in T$,

$$
\left\|x_{1}-x_{2}\right\| \leq\left\|f\left(x_{1}\right)-f\left(x_{2}\right)\right\| \leq(1+\varepsilon)\left\|x_{1}-x_{2}\right\| .
$$

Received by the editors January 15, 2008.

2000 Mathematics Subject Classification. Primary 51M09, 68W40. 
Theorem 1.1 (Dimension Reduction for $H^{n}$ ). Consider the map $g: H^{n} \rightarrow$ $H^{O\left((\log n) / \varepsilon^{2}\right)}$ defined by

$$
g(p) \equiv g((z, x))=(z, f(x)) .
$$

Then for every two points $p_{1}$ and $p_{2}$ at (hyperbolic) distance $\Delta$, we have

$$
\Delta \leq d\left(g\left(p_{1}\right), g\left(p_{2}\right)\right) \leq\left(1+\frac{3 \varepsilon}{1+\Delta}\right) \Delta .
$$

Remark 1.2. Since we reduce the hyperbolic case to the Euclidean case, the dimension reduction embedding for $H^{n}$ can be computed very efficiently using the Fast Johnson-Lindenstrauss Transform of Ailon and Chazelle [1].

The following corollary follows from a result of Bonk and Schramm [2].

Corollary 1.3. Let $X$ be a Gromov hyperbolic geodesic metric space with bounded growth at some scale. Then there exist constants $\lambda_{X}$ and $C_{X}$ such that every $n$ point subset $S$ of $X$ roughly quasi-similar embeds into an $O\left((\log n) / \varepsilon^{2}\right)$ dimensional hyperbolic space. That is, there exists a map $\varphi: S \rightarrow H^{O\left((\log n) / \varepsilon^{2}\right)}$ such that for every $x, y \in S$,

$$
\lambda_{X} d(x, y)-C_{X} \leq d(\varphi(x), \varphi(y)) \leq(1+\varepsilon) \lambda_{X} d(x, y)+C_{X} .
$$

For points far apart we prove the following theorem.

Theorem 1.4 (Embedding into Hyperbolic Plane). Let $S$ be an n-point subset of $H^{n}$. Assume that the distance between every two points in $S$ is at least $\frac{\ln (12 n)}{\varepsilon}$. Then there exists an embedding of $S$ into the hyperbolic plane $H^{2}$ with distortion at most $1+\varepsilon$.

\section{Proofs}

We start with the proof of the first theorem followed by the proof of the second.

Proof of Theorem 1.1. First, since $F_{z_{1}, z_{2}}$ is an increasing function, we have

$$
d\left(g\left(p_{1}\right), g\left(p_{2}\right)\right)=F_{z_{1}, z_{2}}\left(\left\|f\left(x_{1}\right)-f\left(x_{2}\right)\right\|\right) \geq F_{z_{1}, z_{2}}\left(\left\|x_{1}-x_{2}\right\|\right)=\Delta .
$$

On the other hand, by the mean value theorem,

$$
\begin{aligned}
d\left(g\left(p_{1}\right), g\left(p_{2}\right)\right) & \leq F_{z_{1}, z_{2}}\left((1+\varepsilon)\left\|x_{1}-x_{2}\right\|\right) \\
& =F_{z_{1}, z_{2}}\left(\left\|x_{1}-x_{2}\right\|\right)+\frac{d F_{z_{1}, z_{2}}(\hat{r})}{d r} \cdot \varepsilon\left\|x_{1}-x_{2}\right\|,
\end{aligned}
$$

where $\hat{r} \in\left(\left\|x_{1}-x_{2}\right\|,(1+\varepsilon)\left\|x_{1}-x_{2}\right\|\right)$. Let us now bound the derivative of $F_{z_{1}, z_{2}}$ :

$$
\begin{aligned}
\frac{d F_{z_{1}, z_{2}}(\hat{r})}{d r} & =\left.\frac{2 \hat{r}}{2 z_{1} z_{2}} \cdot \frac{1}{\sqrt{t-1} \sqrt{t+1}}\right|_{t=1+\frac{\hat{r}^{2}+\left(z_{1}-z_{2}\right)^{2}}{2 z_{1} z_{2}}} \\
& =\frac{2 \hat{r}}{\sqrt{\hat{r}^{2}+\left|z_{1}-z_{2}\right|^{2}} \sqrt{\hat{r}^{2}+\left|z_{1}-z_{2}\right|^{2}+4 z_{1} z_{2}}} \\
& \leq \frac{2}{\sqrt{\hat{r}^{2}+\left|z_{1}-z_{2}\right|^{2}+4 z_{1} z_{2}}} \\
& \leq \frac{2}{\sqrt{\| x_{1}-\left.x_{2}\right|^{2}+\left|z_{1}-z_{2}\right|^{2}+4 z_{1} z_{2}}} .
\end{aligned}
$$


Here, we used that $(\operatorname{arccosh} t)^{\prime}=1 / \sqrt{(t-1)(t+1)}$. From the identity

$$
\frac{\left\|x_{1}-x_{2}\right\|^{2}+\left|z_{1}-z_{2}\right|^{2}}{2 z_{1} z_{2}}=\cosh \Delta-1,
$$

and the bound for $\frac{d F_{z_{1}, z_{2}}(\hat{r})}{d r}$ we get an estimate for the additive term in (2.2):

$$
\begin{aligned}
\frac{d F_{z_{1}, z_{2}}(\hat{r})}{d r} \cdot \varepsilon\left\|x_{1}-x_{2}\right\| & \leq \frac{2\left\|x_{1}-x_{2}\right\| \varepsilon}{\sqrt{2 z_{1} z_{2}(\cosh \Delta+1)}} \\
& \leq 2 \varepsilon \sqrt{\frac{\left\|x_{1}-x_{2}\right\|^{2}+\left|z_{1}-z_{2}\right|^{2}}{2 z_{1} z_{2}}} \cdot \frac{1}{\sqrt{\cosh \Delta+1}} \\
& =2 \varepsilon \sqrt{\frac{\cosh \Delta-1}{\cosh \Delta+1}}=2 \varepsilon \tanh \frac{\Delta}{2} .
\end{aligned}
$$

It is easy to see that

$$
\tanh t \leq \frac{3 t}{1+2 t}
$$

for $t>0$. Therefore, the additive term in (2.2) is at most

$$
\frac{3 \varepsilon}{1+\Delta} \Delta \text {. }
$$

This concludes the proof.

Proof of Theorem 1.4. Define $T=\{x:(z, x) \in S\}$. By a theorem of Matoušek [6], there exists an embedding $f: T \rightarrow \mathbb{R}$ of $\mathbb{R}^{n-1}$ into $\mathbb{R}$ with distortion at most $12 n$. We assume that $f$ is non-contracting and $\|f\|_{\text {Lip }} \leq 12 n$. Consider the embedding $g: S \rightarrow H^{2}$ defined by

$$
g((z, x))=(z, f(x)) .
$$

Clearly, $g$ is non-contracting. Now we upper bound the Lipschitz norm of $g$. Pick two points $p_{1}=\left(z_{1}, x_{1}\right)$ and $p_{2}=\left(z_{2}, x_{2}\right)$ at distance $\Delta$ in $S$. Let $r=\left\|x_{1}-x_{2}\right\|$.

$$
\begin{aligned}
d\left(g\left(p_{1}\right), g\left(p_{2}\right)\right) & =F_{z_{1}, z_{2}}\left(\left\|f\left(x_{1}\right)-f\left(x_{2}\right)\right\|\right) \leq F_{z_{1}, z_{2}}(12 n r) \\
& \leq \operatorname{arccosh}\left(1+12 n \frac{r^{2}+\left|z_{1}-z_{2}\right|^{2}}{2 z_{1} z_{2}}\right) .
\end{aligned}
$$

Since

we have

$$
\frac{r^{2}+\left|z_{1}-z_{2}\right|^{2}}{2 z_{1} z_{2}}=\cosh \Delta-1,
$$

$$
d\left(g\left(p_{1}\right), g\left(p_{2}\right)\right) \leq \operatorname{arccosh}(12 n \cosh \Delta-(12 n-1))
$$

Observe that

$$
\begin{aligned}
\cosh t & =\frac{e^{t}+e^{-t}}{2} \leq \frac{e^{t}}{2}+\frac{1}{2} \quad(\text { for } t>0) \\
\operatorname{arccosh} t & =\ln \left(t+\sqrt{t^{2}-1}\right) \leq \ln (2 t) \quad(\text { for } t>1)
\end{aligned}
$$

Therefore,

$$
\begin{aligned}
d\left(g\left(p_{1}\right), g\left(p_{2}\right)\right) & \leq \operatorname{arccosh}(12 n \cosh \Delta-(12 n-1)) \leq \ln \left(2 \cdot 12 n \frac{e^{\Delta}}{2}\right) \\
& =\Delta+\ln (12 n) \leq(1+\varepsilon) \Delta .
\end{aligned}
$$

This concludes the proof. 


\section{ACKNOWLEDGEMENTS}

We would like to thank James Lee, Assaf Naor and Oded Schramm for useful discussions.

\section{REFERENCES}

1. N. Ailon and B. Chazelle, Approximate nearest neighbors and the fast Johnson-Lindenstrauss transform, Proc. of the 38th Annual ACM Symposium on Theory of Computing, ACM, New York, 2006, pp. 557-563. MR2277181 (2007h:68074)

2. M. Bonk and O. Schramm, Embeddings of Gromov hyperbolic spaces, Geom. Funct. Anal. 10 (2000), no. 2, 266-306. MR1771428 (2001g:53077)

3. J. Cannon, W. Floyd, R. Kenyon, and W. Parry, Hyperbolic geometry, Flavors of Geometry, Math. Sci. Res. Inst. Publ., 31, Cambridge Univ. Press, Cambridge, 1997, pp. 59-115. MR:1491098(99c:57036)

4. W. B. Johnson and J. Lindenstrauss, Extensions of Lipschitz mappings into a Hilbert space, Contemp. Math., 26, Amer. Math. Soc., Providence, RI (1984), 189-206. MR0737400 (86a:46018)

5. R. Krauthgamer and J. R. Lee, Algorithms on negatively curved spaces, Proc. of the 47th Symposium on Foundations of Computer Science, 2006, pp. 119-132.

6. J. Matoušek. Bi-Lipschitz embeddings into low-dimensional Euclidean spaces, Comment. Math. Univ. Carolin. 31 (1990), no. 3, 589-600. MR1078491 (91k:54056)

Microsoft Research - and - Department of Mathematics, The Weizmann Institute, ReHOVOt 76100, ISRAEL

E-mail address: itai.benjamini@weizmann.ac.il

Microsoft Research New England, One Memorial Drive, Cambridge, Massachusetts 02142

E-mail address: yurym@icrosoft.com 